\title{
An immersed soap film of genus one
}

\author{
ROBERT HUFF
}

In this paper we prove the existence of a set homotopic to a punctured torus and a map from this set into three-space such that each point in the image has an intrinsic soap film neighborhood.

\section{Introduction}

The central cone is the only soap film known to span a regular tetrahedron. Lawlor and Morgan [5] have shown it has least area among all soap films which separate the solid tetrahedron into four regions. If the separation restriction is removed, however, it is not known whether or not the cone is a least area soap film. In fact, it is not known if another soap film spanning a regular tetrahedron even exists. A plausible sketch of a competitor soap film was produced in the mid-1990s by Hardt (see figure 1). The proposed soap film separates the solid tetrahedron into two regions. It consists of two planar disks glued to a punctured torus along $Y$-singularities, which are curves along which three (minimal) surfaces meet in such a way that the angle between any two is $120^{\circ}$. Unlike the cone, this set has no $T$ singularities - points at which four $Y$-singularities meet in such a way that the angle between any two is $\arccos (-1 / 3) \approx 109.47^{\circ}$. As observed by Plateau and proven by Taylor [6], these are the only singularities allowed in a soap film.

In addition to being a competitor to the cone, the object in figure 1 is important because it would be the first example of a soap film with singularities that also has a handle. Specifically, the object in the sketch is homotopic to a punctured torus. While such a soap film seems to fit well with a tetrahedral boundary, all research - both experimental and theoretical - has pointed to non-existence. Thus, in order to prove the existence of a genus one soap film, it may be necessary to modify the boundary. Such a modification is done here (see figure 2), and the result is the following theorem.

Theorem 1.1. There exists a set $\mathcal{X}$ homotopic to a punctured torus and a map $\Phi: \mathcal{X} \rightarrow \mathbb{R}^{3}$ such that each point in $\mathcal{X}$ has a neighborhood $\mathcal{U}$ such that 


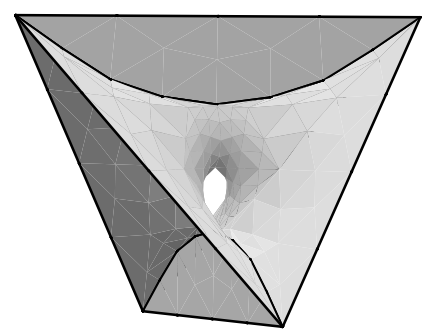

Figure 1: A plausible competitor soap film spanning a regular tetrahedron.

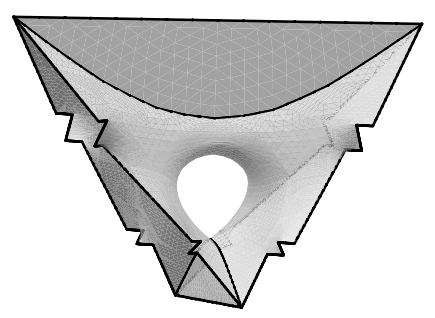

Figure 2: An computer generated sketch of a soap film from Theorem 1.1 spanning a modified tetrahedral boundary.

$\mathcal{X}(\mathcal{U})$ is either a minimal surface or three minimal surfaces meeting along a curve at $120^{\circ}$.

The tetrahedron in figure 1 is oriented in such a way that its top and bottom edges are horizontal. Each of these two edges is contained in the boundary of a planar disk, and the four remaining non-horizontal edges form the boundary of the punctured torus. As a whole, the object in figure 1 has two reflectional symmetries through the planes of the disks. It also has two less obvious symmetries: $180^{\circ}$ rotation around each of the lines through the midpoints of a non-horizontal edge and its adjoint. In the proof of Theorem 1.1, we will assume these same symmetries. We will also keep the two horizontal edges in the boundary, but the four non-horizontal edges will be modified by inserting two "zig-zags" into each. A zig-zag consists of two parallel, horizontal line segments connected by a third, non-horizontal line segment (see figure 3).

\section{Proof of Theorem 1.1}

To prove Theorem 1.1, we take the take viewpoint of Weber and Wolf in [7], where the technique of flat structures is used to prove the existence of 

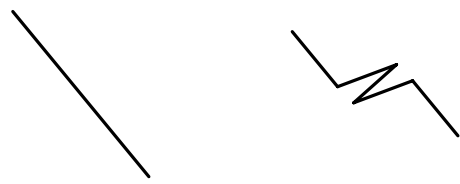

Figure 3: Left: The front, left non-horizontal edge of figure 1. Right: An inserted zig-zag.

complete, properly embedded minimal surfaces of arbitrarily high genus. In that paper, the authors looked for a conformally equivalent pair of domains within a moduli space of pairs which already satisfy conditions guaranteeing the existence of handles. Here, we start with a moduli space of conformally equivalent pairs, and we look for a pair which guarantees a torus.

\subsection{Using the Gauss map to find a parameter domain}

Because of the symmetries described above, the punctured torus in figure 1 consists of eight congruent pieces. Each piece is a disk bounded by four curves (see figure 4):

1. $e_{1}$, which is a curve of mirror symmetry and traverses one-half of a torus-handle.

2. $e_{2}$, which is one-half of a $Y$-singularity.

3. $e_{3}$, which is one-half of a non-horizontal tetrahedral edge.

4. $e_{4}$, which one-half of a rotation axis.

We will choose a piece $\hat{S}$ which can be oriented in $x y x_{3}$-space so that the $Y$-singularity $e_{2}$ lies above the negative $y$-axis in the $y x_{3}$-plane and the torus-handle curve $e_{1}$ lies above the positive $x_{3}$-axis in the $x x_{3}$-plane. This implies the rotation axis $e_{4}$ is horizontal and is parallel to the line $y=-x$. It

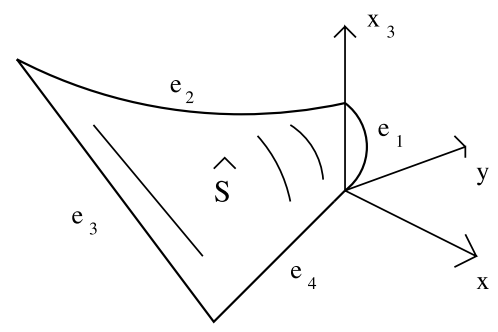

Figure 4: The fundamental piece $\hat{S}$. 
also implies the tetrahedral edge $e_{3}$ is in the direction $\langle-1 / 2,-1 / 2,1 / \sqrt{2}\rangle$. In figure 1 , the piece we are taking is the upper, front, left eighth of the punctured torus.

The surface $\hat{S}$ is a fundamental piece for the punctured torus, and it is also a fundamental piece for the entire soap film. This is because the remaining part of the soap film consists of two planar disks. Thus, the existence of the soap film is implied by the existence of $\hat{S}$. To prove the existence of $\hat{S}$, we will derive a parametrization on its image under the (outward pointing) Gauss map $N$. Based on the properties of $\hat{S}$ discussed so far, we have:

1. Since $e_{1} \subset x x_{3}$-plane is a curve of mirror symmetry, it follows that the Gauss map along $e_{1}$ is also contained in the $x x_{3}$-plane.

2. Since $e_{2} \subset y x_{3}$-plane is a $Y$-singular curve, it follows that $\hat{S}$ meets the $y x_{3}$-plane at a constant angle of $\pi / 3$.

3. The Gauss map along $e_{3}$ is perpendicular to $e_{3}$.

4. The Gauss map along $e_{4}$ is perpendicular to $e_{4}$.

When followed by stereographic projection $\sigma$, the image of $\partial \hat{S}$ in the $x y$-plane has the following properties:

1. $\sigma \circ N\left(e_{1}\right)$ is contained in the $x$-axis.

2. $\sigma \circ N\left(e_{2}\right)$ is contained in the circle $C_{2}=\partial D(2, \sqrt{3})$.

3. $\sigma \circ N\left(e_{3}\right)$ is contained in the circle $C_{3}=\partial D\left(\mathrm{e}^{\mathrm{i} \pi / 4}, \sqrt{2}\right)$.

4. $\sigma \circ N\left(e_{4}\right)$ is contained in the line $y=x$.

Interestingly, these properties are not enough to determine a domain which could reasonably be the Gauss image. This is because the two circles $C_{2}, C_{3}$ and the line $y=x$ have a common intersection point. To get around this issue, we can assume the Gauss map on the soap film oscillates along $e_{3}$. This introduces a branch point somewhere inside the edge and allows us to determine a domain $\Omega$, shown in figure 5 , which we may reasonably assume is the image of the Gauss map under stereographic projection. In particular, the domain $\Omega$ consists of all points in the upper half plane that lie below the line $y=x$ and to the left of the rightmost arc of $C_{2}$ connecting the line $y=x$ with the $x$-axis, minus points on some arc of $C_{3}$ emanating from the line $y=x$ with a downward trajectory. This arc of $C_{3}$ has two important properties. First, it should be thought of as having two sides $e_{3}^{l}$ and $e_{3}^{\mathrm{u}}$. As $z \in \Omega$ approaches the arc from the inside of $C_{3}$, the image under 


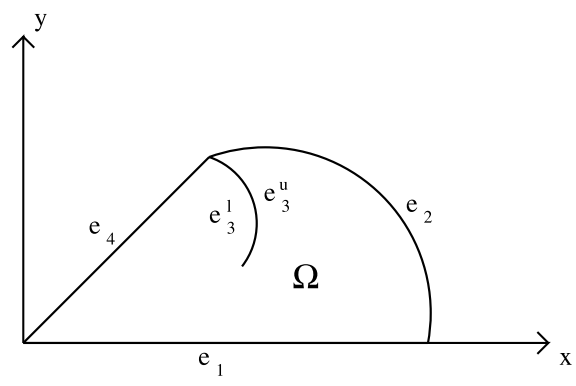

Figure 5: The domain $\Omega$.

the yet-to-be-derived parametrization approaches the bottom of the edge $e_{3}$ on $\hat{S}$. Similarly, as $z$ approaches the arc from outside of $C_{3}$ the image under the parametrization approaches the upper portion of $e_{3}$. Secondly, the length of this arc is undetermined since there is no reason to specify the extent of the assumed oscillation of the Gauss map along $e_{3}$. However, this is actually helpful since we will use this freedom to solve what is called a "period problem" on the curve $e_{1}$.

\subsection{Determining the developed image of the square root of the second fundamental form}

We wish to prove the existence of $\hat{S}$ by deriving a parametrization for such a surface on the domain $\Omega$. Since $\hat{S}$ is to be minimal, it will be useful to apply the following application of the Weierstrass Representation Theorem for minimal surfaces.

Theorem 2.1. Let $\Omega \subset \mathbb{C}$ be simply connected. If $g$ is a meromorphic function and $d h$ is a holomorphic one-form on $\Omega$ which are compatible in the sense that $g$ has a zero or pole of order $n$ at $p \in \Omega$ if and only if dh has a zero of order $n$ at $p \in \Omega$, then the map $X=\left(X_{1}, X_{2}, X_{3}\right): \Omega \rightarrow \mathbb{R}^{3}$ given by

$$
X(z)=\operatorname{Re} \int^{z}\left(\frac{1}{2}\left(g^{-1}-g\right), \frac{\mathrm{i}}{2}\left(g^{-1}+g\right), 1\right) d h
$$

is a conformal, minimal immersion. Moreover, the function $g$ is stereographic projection of the Gauss map on the surface.

In our case, we have assumed the domain $\Omega$ is the image of the desired surface under stereographic projection of the Gauss map. Thus, we may assume $g(z)=z$. The second piece of data is the one form $d h$, which is a 
holomorphic extension of $d X_{3}$ and is called the complexified height differential. To derive this, we will use a formula that relates the second fundamental form $I I$ on a minimal surface to the Weierstrass data $g$ and $d h$. In particular, for vectors $\mathbf{v}$ and $\mathbf{w}$ in the tangent plane to the surface at a point, we have

$$
\frac{d g(\mathbf{v}) d h(\mathbf{w})}{g}=I I(\mathbf{v}, \mathbf{w})-\mathrm{i} I I(\mathbf{v}, \mathrm{iw}) .
$$

From (2.2) it follows that:

$$
c \text { is a principal curve } \Leftrightarrow \frac{d g(\dot{c}) d h(\dot{c})}{g} \in \mathbb{R}
$$

and

$$
c \text { is an asymptotic curve } \Leftrightarrow \frac{d g(\dot{c}) d h(\dot{c})}{g} \in i \mathbb{R} .
$$

A nice proof of formula (2.2) as well as the statements of properties (2.3) and $(2.4)$ can be found in [4].

We see from (2.3) and (2.4) that the function $\zeta$ given by

$$
\zeta(z)=\int^{z} \sqrt{\frac{d g d h}{g}}
$$

maps principal curves into vertical or horizontal lines in $\mathbb{C}$ and asymptotic curves into lines in one of the directions $\mathrm{e}^{ \pm \mathrm{i} \pi / 4}$. The map $\zeta$ is called the developing map of the one form $\sqrt{\frac{d g d h}{g}}$. It is a local isometry between the minimal surface equipped with the conformal cone metric $\left|\frac{d g d h}{g}\right|$ and $\mathbb{C}$ equipped with the Euclidean metric.

On the surface $\hat{S}$, the line segments $e_{3}$ and $e_{4}$ are clearly asymptotic curves. For each of the curves $e_{1}$ and $e_{2}$, we have from the properties listed above that the surface meets the plane of the curve at a constant angle. Thus, from Joachimstahl's theorem (see [2]) it follows these two curves are principal. Finally, we must deal with the introduction of a branch point inside $e_{3}$. The effect of this on $\Omega$ was to produce a five sided curvilinear domain with two edges $e_{3}^{1}$ and $e_{3}^{\mathrm{u}}$ comprising the Gauss image of $e_{3}$ on $\hat{S}$. Since the angle between $e_{3}^{1}$ and $e_{3}^{\mathrm{u}}$ is $2 \pi$ and the angle between their images under the parametrization into $\mathbb{R}^{3}$ should be $\pi$, it follows that the angle 

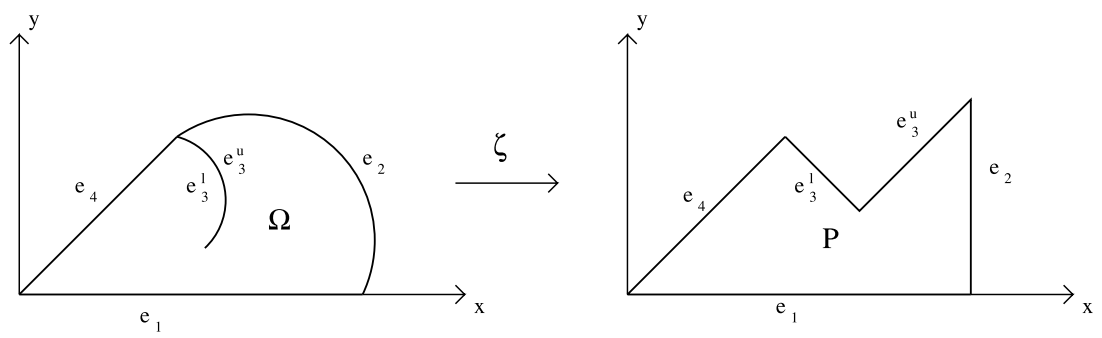

Figure 6: The image of $\Omega$ under $\zeta$.

between their images under $\zeta$ should be $3 \pi / 2$. Thus, we conclude the image

$$
P=\zeta(\Omega)
$$

should be a Euclidean pentagon as shown in figure 6. In particular, this polygon should be such that

1. $\zeta\left(e_{1}\right)$ is parallel to the $x$-axis.

2. $\zeta\left(e_{2}\right)$ is parallel to the $y$-axis.

3. $\zeta\left(e_{3}^{\mathrm{u}}\right)$ and $\zeta\left(e_{4}\right)$ are parallel to the line $y=x$.

4. $\zeta\left(e_{3}^{1}\right)$ is parallel to the line $y=-x$.

Furthermore, we can normalize $P$ so that the vertex $v_{14}=e_{1} \cap e_{4}$ is the origin and the edge $e_{1}$ has unit length.

We have thus derived a minimal immersion

$$
X=\left(X_{1}, X_{2}, X_{3}\right): \Omega \rightarrow \mathbb{R}^{3},
$$

with the formula for $X$ given by Equation (2.1). Here, we have

$$
g(z)=z \quad \text { and } \quad d h=\frac{g(d \zeta)^{2}}{d g},
$$

and we assume the base point of integration is the origin so that

$$
X\left(v_{14}\right)=(0,0,0) .
$$

However, we have yet to prove that such a map $\zeta$ exists. 


\subsection{Existence of the map $\zeta$}

The map $\zeta$ is an edge preserving conformal map between the domains $\Omega$ and $P$. The existence of a conformal map between the domains is guaranteed by the Riemann mapping theorem, but the edge preserving property is not. For this, we need the conformal invariant extremal length. We will restrict our attention to curvilinear polygons, although in general extremal length is defined on arbitrary domains.

Given a curvilinear polygon $\Delta$, a Borel measurable function $\rho>0$ on $\Delta$ defines a conformal metric $\rho\left(d x^{2}+d y^{2}\right)$. The length of a curve $\gamma \subset \Delta$ with respect to $\rho$ is denoted $\ell_{\rho}(\gamma)$ (with $|\gamma|$ denoting Euclidean length), and the $\rho$-area of $\Delta$ is denoted by $A_{\rho}$. With this notation, we define the extremal length between edges $A$ and $B$ by

$$
\operatorname{Ext}_{\Delta}(A, B)=\sup _{\rho} \frac{\inf _{\gamma} \ell_{\rho}^{2}(\gamma)}{A_{\rho}}
$$

where the infimum is taken over all curves $\gamma:[0,1] \rightarrow \Delta$ such that $\gamma(0) \in A$, $\gamma(1) \in B$, and $\gamma(t) \in \operatorname{interior}(\Delta)$ for $t \in(0,1)$. Extremal length is invariant under biholomorphisms and has the following properties, which we record here (for more details, see [1]).

Proposition 2.1. $\quad$ (i) Extremal length depends continuously on $\Delta, A$ and $B$.

(ii) If $A$ and $B$ are adjacent edges, then $\operatorname{Ext}_{\Delta}(A, B)=0$

(iii) If $B$ is degenerate (i.e., $B$ is a point) and $\operatorname{dist}(A, B)>0$, then $\operatorname{Ext}_{\Delta}(A, B)=\infty$

(iv) If $\Delta_{1} \subset \Delta_{2}$ are such that edges $A_{k}, B_{k} \subset \Delta_{k}, k=1,2$, satisfy $A_{1} \subset A_{2}$ and $B_{1} \subset B_{2}$, then

$$
\operatorname{Ext}_{\Delta_{2}}\left(A_{2}, B_{2}\right) \leq \operatorname{Ext}_{\Delta_{1}}\left(A_{1}, B_{1}\right)
$$

where the inequality is strict if $\operatorname{dist}\left(A_{2}, B_{2}\right)>0$ and either $A_{1} \neq A_{2}$ or $B_{1} \neq B_{2}$.

We are now ready to prove the following proposition.

Proposition 2.2. There exists an edge-preserving conformal map $\zeta$ from $\Omega$ onto some Euclidean pentagon $P$ with edges oriented and labeled as in figure 6 . 
Proof. Consider a Euclidean pentagon $P$ with edges oriented and labeled as in figure 6 and normalized so that $v_{14}=0$ and $\left|e_{1}\right|=1$. Any such pentagon is determined by the lengths $\ell=\left|e_{2}\right|$ and $m=\left|e_{3}^{\mathrm{u}}\right|$, where the set of possible $(\ell, m)$ pairs is

$$
\mathcal{P}=\{(\ell, m) \mid 0<\ell<1 \text { and } 0<m<\ell \sqrt{2}\} .
$$

So, we can write $P=P_{\ell m}$.

If we fix $\ell$ while allowing $m$ to vary, it follows from parts (i), (ii) and (iii) of Proposition 2.1 that:

$$
\operatorname{Ext}_{P_{\ell m}}\left(e_{1}, e_{3}^{\mathrm{u}}\right) \rightarrow 0 \text { as } m \rightarrow \ell \sqrt{2}
$$

and

$$
\operatorname{Ext}_{P_{\ell m}}\left(e_{1}, e_{3}^{\mathrm{u}}\right) \rightarrow \infty \text { as } m \rightarrow 0 .
$$

Therefore, by continuity there exists some intermediate $\hat{m}=f_{1}(\ell)$ such that

$$
\operatorname{Ext}_{\Omega}\left(e_{1}, e_{3}^{\mathrm{u}}\right)=\operatorname{Ext}_{P_{\ell \hat{m}}}\left(e_{1}, e_{3}^{\mathrm{u}}\right) .
$$

The value $f_{1}(\ell)$ must be bounded away from zero as $\ell$ approaches 1 (see figure 7 ), since otherwise we would have

$$
\operatorname{Ext}_{P_{\ell \dot{m}}}\left(e_{1}, e_{3}^{\mathrm{u}}\right) \rightarrow \infty \text { as } \ell \rightarrow 1 .
$$

Also, we have that $f_{1}(\ell)$ must be bounded away from $\sqrt{2}$ as $\ell$ approaches 1 , since otherwise it would follow that

$$
\operatorname{Ext}_{P_{\ell \hat{m}}}\left(e_{1}, e_{3}^{\mathrm{u}}\right) \rightarrow 0 \text { as } \ell \rightarrow 1 .
$$

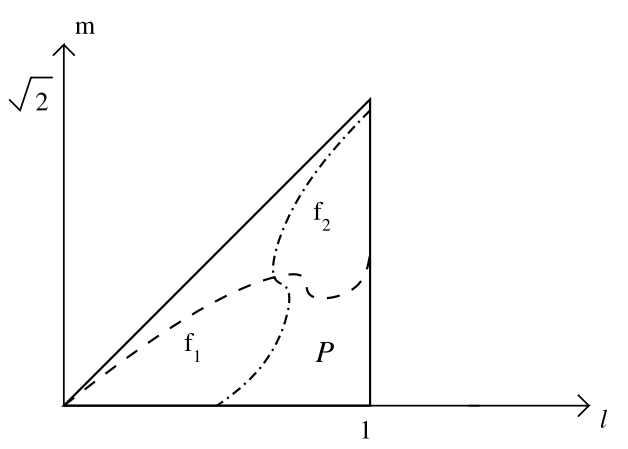

Figure 7: The graphs of $f_{1}$ and $f_{2}$ in the $\ell m$-plane must intersect. 
Next, fix $m$ and let $\ell$ vary. Arguing as before, it follows that:

$$
\operatorname{Ext}_{P_{\ell m}}\left(e_{1}, e_{3}^{1}\right) \rightarrow 0 \text { as } \ell \rightarrow \frac{m}{\sqrt{2}}
$$

and

$$
\operatorname{Ext}_{P_{\ell m}}\left(e_{1}, e_{3}^{1}\right) \rightarrow \infty \text { as } \ell \rightarrow 1 .
$$

Thus, there is an intermediate $\hat{\ell}=f_{2}(m)$ such that

$$
\operatorname{Ext}_{\Omega}\left(e_{1}, e_{3}^{1}\right)=\operatorname{Ext}_{P_{\hat{\ell} m}}\left(e_{1}, e_{3}^{1}\right) .
$$

We have as before that $f_{2}(m)$ must be bounded away from zero as $m$ approaches zero, since otherwise we would have

$$
\operatorname{Ext}_{P_{\hat{\ell} m}}\left(e_{1}, e_{3}^{1}\right) \rightarrow 0 \text { as } m \rightarrow 0 .
$$

Furthermore, it must be true that $f_{2}(m)$ is bounded away from 1 as $m$ approaches zero, since otherwise it would follow that

$$
\operatorname{Ext}_{P_{\hat{\ell} m}}\left(e_{1}, e_{3}^{1}\right) \rightarrow \infty \text { as } m \rightarrow 0 .
$$

It follows from $(2.8),(2.9),(2.11)$ and (2.12) that the two graphs intersect, and so there is some pentagon $\hat{P}=P_{\hat{\ell} \hat{m}}$ such that (2.7) and (2.10) are both satisfied. We now show this is the desired $P$ of the proposition.

By the Riemann mapping theorem, there exists a conformal map $\zeta$ from $\Omega$ onto $\hat{P}$, and we can normalize so that

$$
\zeta\left(v_{12}\right)=v_{12}, \zeta\left(v_{14}\right)=v_{14} \quad \text { and } \quad \zeta\left(v_{34}\right)=v_{34},
$$

where

$$
v_{12}=e_{1} \cap e_{2} \quad \text { and } \quad v_{34}=e_{3}^{1} \cap e_{4} .
$$

Moreover, since (2.10) holds, it follows from statement (iv) of Proposition 2.1 that

$$
\zeta\left(v_{3}\right)=v_{3},
$$

where

$$
v_{3}=e_{3}^{\mathrm{l}} \cap e_{3}^{\mathrm{u}} .
$$

Similarly, given (2.14), it follows from (2.7) that

$$
\zeta\left(v_{23}\right)=v_{23},
$$


where

$$
v_{23}=e_{2} \cap e_{3}^{\mathrm{u}}
$$

Therefore, from (2.13), (2.14) and (2.15) we have that $\zeta$ is the desired conformal, edge-preserving map of the proposition.

\subsection{Verification of the parametrization}

To show that the image $X(\Omega)$ is indeed a surface as in figure 4 , we first need to make sure its closure is compact. To accomplish this, we note from the descriptions of $\Omega$ and $P=\zeta(\Omega)$ that

1. At $v_{14}$ on $\partial \Omega$, the map $\zeta$ takes an angle of $\pi / 4$ to an angle of $\pi / 4$ on $P$.

2. At $v_{12}$ on $\partial \Omega$, the map $\zeta$ takes an angle of $\pi / 2$ to an angle of $\pi / 2$ on $P$.

3. At $v_{23}$ on $\partial \Omega$, the map $\zeta$ takes the angle $\phi_{23}=\arccos (1 / \sqrt{3}) \approx 54.7^{\circ}$ to an angle of $\pi / 4$ on $P$.

4. At $v_{3}$ on $\partial \Omega$, the map $\zeta$ takes an angle of $2 \pi$ to an angle of $3 \pi / 2$ on $P$.

5. At $v_{34}$ on $\partial \Omega$, the map $\zeta$ takes an angle of $\pi / 2$ to an angle of $\pi / 2$ on $P$.

The above properties imply

$$
d h=\frac{g(d \zeta)^{2}}{d g}=z \zeta^{\prime}(z)^{2} d z
$$

is integrable on $\Omega$-neighborhoods of the vertices, which implies

$$
\overline{X(\Omega)} \text { is compact. }
$$

Next, we analyze $X$ on $\partial \Omega$ to ensure the boundary of the image in $\mathbb{R}^{3}$ has the geometric properties we expect. Beginning with $e_{1}$, we parameterize from $v_{14}=0$ to $v_{12}=2+\sqrt{3}$ by

$$
z_{1}(w)=w, \quad 0<w<2+\sqrt{3}
$$


Here, we have $d z\left(\dot{z}_{1}\right) \equiv 1$ and $d \zeta\left(\dot{z}_{1}\right)^{2}>0$. Computing, we have

$$
\begin{aligned}
d X_{1}\left(\dot{z}_{1}\right) & =\operatorname{Re}\left(\frac{1}{2}\left(\left(1-z_{1}^{2}\right) \frac{d \zeta\left(\dot{z}_{1}\right)^{2}}{d z\left(\dot{z}_{1}\right)}\right)\right) \\
& =\frac{d \zeta\left(\dot{z}_{1}\right)^{2}}{2}\left(1-w^{2}\right) .
\end{aligned}
$$

Continuing, we have

$$
\begin{aligned}
d X_{2}\left(\dot{z}_{1}\right) & =\operatorname{Re}\left(\frac{1}{2}\left(\mathrm{i}\left(1+z_{1}^{2}\right) \frac{d \zeta\left(\dot{z}_{1}\right)^{2}}{d z\left(\dot{z}_{1}\right)}\right)\right) \\
& =\frac{d \zeta\left(\dot{z}_{1}\right)^{2}}{2} \operatorname{Re}\left(\mathrm{i}\left(1+w^{2}\right)\right)=0 .
\end{aligned}
$$

For the $x_{3}$ component, we have

$$
\begin{aligned}
d X_{3}\left(\dot{z}_{1}\right) & =\operatorname{Re}\left(\frac{z_{1} d \zeta\left(\dot{z}_{1}\right)^{2}}{d z\left(\dot{z}_{1}\right)}\right) \\
& =d \zeta\left(\dot{z}_{1}\right)^{2} w>0 .
\end{aligned}
$$

The Gauss map along $e_{1}$ as well as Equations (2.17) to (2.19) imply $X\left(e_{1}\right)$ is a curve of mirror symmetry in the $x x_{3}$-plane which is the graph of some function $h_{1}$ over the $x_{3}$-axis, where $X_{1}=h_{1}\left(X_{3}\right)$. Furthermore, we can compute

$$
h_{1}^{\prime}\left(X_{3}\right)=\frac{\left(X_{1}\right)^{\prime}}{\left(X_{3}\right)^{\prime}}=\frac{1-w^{2}}{2 w}
$$

and so

$$
h_{1}^{\prime \prime}\left(X_{3}\right)=\frac{\left(\left(X_{3}\right)^{\prime} /\left(X_{1}\right)^{\prime}\right)^{\prime}}{\left(X_{3}\right)^{\prime}}=-\frac{2\left(1+w^{2}\right)}{4 w^{2}\left(X_{3}\right)^{\prime}}<0
$$

Therefore, we have shown

The graph of $h_{1}$ is concave downward.

We next parameterize $e_{2}$ in the counterclockwise direction from $v_{12}$ to $v_{23}$ by $z_{2}(w)=2+\sqrt{3} e^{\mathrm{i} w}, 0<w<\arccos ((1-\sqrt{2}) / \sqrt{6}) \approx 99.7^{\circ}$. Here, we 
have $d z\left(\dot{z}_{2}\right)=i \sqrt{3} \mathrm{e}^{\mathrm{i} w}$ and $d \zeta\left(\dot{z}_{2}\right)^{2}<0$. Calculating, we have

$$
\begin{aligned}
d X_{1}\left(\dot{z}_{2}\right) & =\operatorname{Re} \frac{1}{2}\left(\left(1-z_{2}^{2}\right) \frac{d \zeta\left(\dot{z}_{2}\right)^{2}}{d z\left(\dot{z}_{2}\right)}\right) \\
& =-\frac{d \zeta\left(\dot{z}_{2}\right)^{2}}{2 \sqrt{3}} \operatorname{Re}\left(\mathrm{ie}^{-\mathrm{i} w}\left(-3-4 \sqrt{3} \mathrm{e}^{\mathrm{i} w}-3 \mathrm{e}^{\mathrm{i} 2 w}\right)\right)=0 .
\end{aligned}
$$

Continuing, we have

$$
\begin{aligned}
d X_{2}\left(\dot{z}_{2}\right) & =\operatorname{Re} \frac{1}{2}\left(\mathrm{i}\left(1+z_{2}^{2}\right) \frac{d \zeta\left(\dot{z}_{2}\right)^{2}}{d z\left(\dot{z}_{2}\right)}\right) \\
& =\frac{d \zeta\left(\dot{z}_{2}\right)^{2}}{2 \sqrt{3}} \operatorname{Re}\left(\mathrm{e}^{-\mathrm{i} w}\left(5+4 \sqrt{3} \mathrm{e}^{\mathrm{i} w}+3 \mathrm{e}^{\mathrm{i} 2 w}\right)\right) \\
& =\frac{2 d \zeta\left(\dot{z}_{2}\right)^{2}}{\sqrt{3}}(2 \cos w+\sqrt{3})<0
\end{aligned}
$$

The reason the inequality is true is because $2 \cos w+\sqrt{3}$ is positive for $-5 \pi / 6<w<5 \pi / 6$. For the $x_{3}$ component, we have

$$
\begin{aligned}
d X_{3}\left(\dot{z}_{2}\right) & =\operatorname{Re}\left(\frac{z_{2} d \zeta\left(\dot{z}_{2}\right)^{2}}{d z\left(\dot{z}_{2}\right)}\right) \\
& =-\frac{d \zeta\left(\dot{z}_{2}\right)^{2}}{\sqrt{3}} \operatorname{Re}\left(\mathrm{ie}^{-\mathrm{i} w}\left(2+\sqrt{3} \mathrm{e}^{\mathrm{i} w}\right)\right)=-\frac{2 d \zeta\left(\dot{z}_{2}\right)^{2}}{\sqrt{3}}(\sin w)>0
\end{aligned}
$$

Equations (2.22) to (2.24) imply $X\left(e_{2}\right)$ is the graph of some decreasing function $h_{2}$ in a plane parallel to the $y x_{3}$-plane, where $X_{3}=h_{2}\left(X_{2}\right)$. Furthermore, we can compute

$$
h_{2}^{\prime}\left(X_{2}\right)=\frac{\left(X_{3}\right)^{\prime}}{\left(X_{2}\right)^{\prime}}=-\frac{\sin w}{2 \cos w+\sqrt{3}},
$$

and so

$$
h_{2}^{\prime \prime}\left(X_{2}\right)=\frac{\left(\left(X_{3}\right)^{\prime} /\left(X_{2}\right)^{\prime}\right)^{\prime}}{\left(X_{2}\right)^{\prime}}=-\frac{2+\sqrt{3} \cos w}{(2 \cos w+\sqrt{3})^{2}\left(X_{2}\right)^{\prime}}>0
$$

Therefore, we have shown

$$
X\left(e_{2}\right) \text { is the graph of a decreasing, }
$$

(2.26) concave upward function in a plane parallel to the $y x_{3}-$ plane. 
Additionally, we have that the outward pointing Gauss map at each point of $X\left(e_{2}\right)$ has positive $x_{3}$ component. Thus, if we consider at each point the tangent vector with positive $x$-component which is perpendicular to the curve $X\left(e_{2}\right)$, then the angle between this tangent vector and its image under reflection through the $y x_{3}$-plane is $120^{\circ}$. This property implies $X\left(e_{2}\right)$ will be a $Y$-singularity if we can extend the surface $X(\Omega)$ to a soap film.

Next, we parameterize $e_{3}^{\mathrm{u}}$ in the counterclockwise direction from $v_{3}$ to $v_{23}$ by

$$
z_{3}(w)=\mathrm{e}^{\mathrm{i} \pi / 4}+\sqrt{2} \mathrm{e}^{\mathrm{i} w}, \quad \theta<w<\pi / 4,
$$

where $\theta$ corresponds to $v_{3}$ and is bounded below by $-\pi / 6$. Here, we have $\dot{z}_{3}(w)=\mathrm{i} \sqrt{2} \mathrm{e}^{\mathrm{i} w}$ and $d \zeta\left(\dot{z}_{3}\right)^{2}=i\left|d \zeta\left(\dot{z}_{3}\right)\right|^{2}$. Computing, we have

$$
\begin{aligned}
d X_{1}\left(\dot{z}_{3}\right) & =\frac{\left|d \zeta\left(\dot{z}_{3}\right)\right|^{2}}{2 \sqrt{2}} \operatorname{Re}\left(\mathrm{e}^{-\mathrm{i} w}\left(1-\mathrm{i}-2 \sqrt{2} \mathrm{e}^{\mathrm{i}(\pi / 4+w)}-2 \mathrm{e}^{\mathrm{i} 2 w}\right)\right) \\
& =-\frac{\left|d \zeta\left(\dot{z}_{3}\right)\right|^{2}}{2 \sqrt{2}}(2+\cos w+\sin w)<0 .
\end{aligned}
$$

Continuing, we have

$$
\begin{aligned}
d X_{2}\left(\dot{z}_{3}\right) & =\frac{\left|d \zeta\left(\dot{z}_{3}\right)\right|^{2}}{2 \sqrt{2}} \operatorname{Re}\left(\mathrm{ie}^{-\mathrm{i} w}\left(1+\mathrm{i}+2 \sqrt{2} \mathrm{e}^{\mathrm{i}(\pi / 4+w)}+2 \mathrm{e}^{\mathrm{i} 2 w}\right)\right) \\
& =-\frac{\left|d \zeta\left(\dot{z}_{3}\right)\right|^{2}}{2 \sqrt{2}}(2+\cos w+\sin w)<0
\end{aligned}
$$

For the $x_{3}$ component, we have

$$
\begin{aligned}
d X_{3}\left(\dot{z}_{3}\right) & =\frac{\left|d \zeta\left(\dot{z}_{3}\right)\right|^{2}}{\sqrt{2}} \operatorname{Re}\left(\mathrm{e}^{-\mathrm{i} w}\left(\mathrm{e}^{\mathrm{i} \pi / 4}+\sqrt{2} \mathrm{e}^{\mathrm{i} w}\right)\right) \\
& =\frac{\left|d \zeta\left(\dot{z}_{3}\right)\right|^{2}}{2}(2+\cos w+\sin w)>0 .
\end{aligned}
$$

From Equations (2.28) to (2.30) we have that the vector $d X$ is given by

$$
\begin{aligned}
\left\langle d X_{1}\left(\dot{z}_{3}\right), d X_{2}\left(\dot{z}_{3}\right), d X_{3}\left(\dot{z}_{3}\right)\right\rangle= & \frac{\left|d \zeta\left(\dot{z}_{3}\right)\right|^{2}}{\sqrt{2}}(2+\cos w+\sin w) \\
& \langle-1 / 2,-1 / 2,1 / \sqrt{2}\rangle .
\end{aligned}
$$

If we parameterize $e_{3}^{1}$ in the counterclockwise direction, then the parametrization is the same as for $e_{3}^{\mathrm{u}}$ but we have $d \zeta^{2}=-i|d \zeta|^{2}$. So, the 
calculations in this case will give

$$
d X=-\frac{|d \zeta|^{2}}{\sqrt{2}}(2+\cos w+\sin w)\langle-1 / 2,-1 / 2,1 / \sqrt{2}\rangle .
$$

Thus, together with the findings for $e_{3}^{\mathrm{u}}$ we have that $X$ maps $e_{3}^{\mathrm{l}} \cup e_{3}^{\mathrm{u}}$ monotonically onto a line segment in the desired direction $\langle-1 / 2,-1 / 2,1 / \sqrt{2}\rangle$.

Finally, we parameterize $e_{4}$ from $v_{14}=0$ to $v_{34}$ by $z_{4}(w)=w \mathrm{e}^{\mathrm{i} \pi / 4}, 0<$ $w<1+\sqrt{2}$. Here, we have $\dot{z}_{4}(w)=\mathrm{e}^{\mathrm{i} \pi / 4}$ and $d \zeta\left(\dot{z}_{4}\right)^{2}=\mathrm{i}\left|d \zeta\left(\dot{z}_{4}\right)\right|^{2}$. Computing, we obtain

$$
\begin{aligned}
d X_{1}\left(\dot{z}_{4}\right) & =\frac{\left|d \zeta\left(\dot{z}_{4}\right)\right|^{2}}{2} \operatorname{Re}\left(\mathrm{i} e^{-\mathrm{i} \pi / 4}\left(1-\mathrm{i} w^{2}\right)\right) \\
& =\frac{\left|d \zeta\left(\dot{z}_{4}\right)\right|^{2}}{2 \sqrt{2}}\left(1+w^{2}\right)>0
\end{aligned}
$$

Continuing, we have

$$
\begin{aligned}
d X_{2}\left(\dot{z}_{4}\right) & =-\frac{\left|d \zeta\left(\dot{z}_{4}\right)\right|^{2}}{2} \operatorname{Re}\left(\mathrm{e}^{-\mathrm{i} \pi / 4}\left(1+\mathrm{i} w^{2}\right)\right) \\
& =-\frac{\left|d \zeta\left(\dot{z}_{4}\right)\right|^{2}}{2 \sqrt{2}}\left(1+w^{2}\right)<0
\end{aligned}
$$

For the $x_{3}$ component, we have

$$
d X_{3}\left(\dot{z}_{4}\right)=\left|d \zeta\left(\dot{z}_{4}\right)\right|^{2} \operatorname{Re}(\mathrm{i} w)=0
$$

From Equations (2.31) to (2.33) we have that the vector $d X$ is given by

$$
\left\langle d X_{1}\left(\dot{z}_{4}\right), d X_{2}\left(\dot{z}_{4}\right), d X_{3}\left(\dot{z}_{4}\right)\right\rangle=\frac{\left|d \zeta\left(\dot{z}_{4}\right)\right|^{2}}{2 \sqrt{2}}\left(1+w^{2}\right)\langle 1,-1,0\rangle .
$$

Thus, we have shown that $X$ maps $e_{4}$ monotonically onto a line segment in the desired direction $\langle 1,-1,0\rangle$.

\subsection{The period condition}

In the preceding subsection, we verified that the boundary of $X(\Omega)$ consists of the curves expected from figure 4. One thing that was not verified, however, was that the curve $X\left(e_{1}\right)$ satisfies a certain "period condition" which ensures we actually get a torus handle when we extend the surface 
by applying the reflectional and rotational symmetries described above. In particular, since $X_{1}\left(v_{14}\right)=0$, we must also have that

$$
X_{1}\left(v_{12}\right)=0 .
$$

In general this condition will not be satisfied, but we will show there are cases where it is. To do this, it is helpful to notice that

$$
\Omega=\Omega_{\theta},
$$

where $\theta$ is the parameter introduced in Equation (2.27). As shown above, this parameter can assume any value strictly between $-\pi / 6$ and $\pi / 4$. Thus, we actually have a family of parameterizations

$$
X^{\theta}=\left(X_{1}^{\theta}, X_{2}^{\theta}, X_{3}^{\theta}\right)
$$

given by Weierstrass data

$$
g(z)=z \quad \text { and } \quad d h=\frac{g d \zeta_{\theta}^{2}}{d g},
$$

where $\zeta_{\theta}$ is a conformal, edge preserving map from $\Omega_{\theta}$ onto a Euclidean pentagon $P_{\theta}$ with edges oriented and labeled as in figure 6 . Notice that $\zeta_{\theta}$ exists for each value of $\theta$ since the proof of Proposition 2.2 does not depend on $\theta$. The period condition can now be written as

$$
X_{1}^{\theta}\left(v_{12}\right)=0 .
$$

As an integral, this takes the form

$$
\Pi(\theta)=X_{1}^{\theta}\left(v_{12}\right)=\frac{1}{2} \int_{0}^{2+\sqrt{3}}\left(1-w^{2}\right) \zeta_{\theta}^{\prime}(w)^{2} d w,
$$

and the period condition is satisfied if

$$
\Pi(\theta)=0 .
$$

Here, we note that $\Pi$ is continuous since $\zeta_{\theta}^{\prime}$ varies continuously with $\theta$ on the edge $e_{1}$.

2.5.1. Negative period We will first consider the case where $\theta \rightarrow-\pi / 6$, which means $v_{3}$ approaches the $x$-axis. Specifically, we will prove Proposition 2.3 below. To do this we will need the following version of the Carathéodory Kernel Convergence Theorem (see [3]). 
Theorem 2.2. Suppose $\Omega$ is a domain in $\mathbb{C}$ bounded by a Jordan curve and $p$ is a point in the interior of $\Omega$. If $\left\{f_{n}\right\}$ is a sequence of univalent functions such that $f_{n}(p)=f_{n+1}(p)$ for all $n$ and $f^{\prime}(p)>0$, then $\left\{f_{n}\right\}$ converges locally uniformly on $\Omega$ if and only if the domains $f_{n}(\Omega)$ converge to a domain $\mathcal{F}$. In the case of convergence, the limit function of $\left\{f_{n}\right\}$ maps $\Omega$ onto $\mathcal{F}$.

Proposition 2.3. There is a value $-\pi / 6<\hat{\theta}<\pi / 4$ such that $\Pi(\hat{\theta})<0$.

Proof. First of all, note that

$$
\operatorname{Ext}_{\Omega_{\theta}}\left(e_{3}^{\mathrm{u}}, e_{1}\right) \rightarrow 0 \text { as } \theta \rightarrow-\pi / 6
$$

and so the corresponding behavior in $P_{\theta}$ must be that the vertex $\zeta_{\theta}\left(v_{3}\right)$ approaches the edge $e_{1}$. Thus, because of compactness there is a sequence $\theta_{n} \rightarrow-\pi / 6$ and a point $0 \leq p \leq 1$ such that

$$
\zeta_{\theta_{n}}\left(v_{3}\right) \rightarrow p \text { as } n \rightarrow \infty
$$

Let $P_{-\pi / 6}$ be the (degenerate) Euclidean polygon with edges oriented and labeled as in figure 6 that is determined by the normalizations $e_{1} \cap e_{4}=$ $0,\left|e_{1}\right|=1$ and the point $p$. From figure 8 , we see $P_{-\pi / 6}$ consists of two triangles

$$
P_{-\pi / 6}^{1} \text { and } P_{-\pi / 6}^{2},
$$

where $P_{-\pi / 6}=P_{-\pi / 6}^{1}$ if $p=1$ and $P_{-\pi / 6}=P_{-\pi / 6}^{2}$ if $p=0$. To apply Theorem 2.2 , we separate $\Omega_{\theta},-\pi / 6<\theta<\pi / 4$ into two (fixed) regions $\Omega^{1}$ and $\Omega^{2}$ via the arc $A_{\theta}$ of $C_{3}$ connecting $v_{3}$ to the point $V_{-\pi / 6}=\frac{1+\sqrt{3}}{\sqrt{2}}$ on $e_{1}$ (see figure 9 ). The domain $\Omega^{1}$ can be extended via reflection around the origin to a domain $\hat{\Omega}^{1}$ bounded by a Jordan curve. The corresponding reflections around the

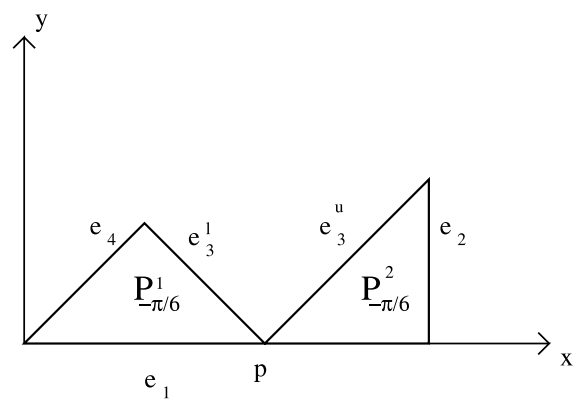

Figure 8: A possible limit polygon $P_{-\pi / 6}$. 

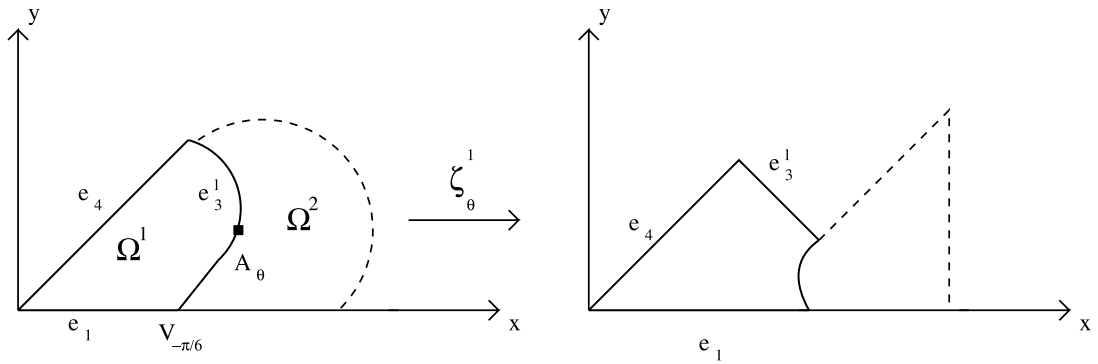

Figure 9: Restriction of $\zeta_{\theta}$ to $\Omega_{\theta}^{1}$.

origin for $\zeta_{\theta_{n}}^{1}\left(\Omega^{1}\right)$, where $\zeta_{\theta_{n}}^{j}$ denotes the restriction of $\zeta_{\theta_{n}}$ to $\Omega^{j}$, extend each map $\zeta_{\theta_{n}}^{1}$ via the Schwarz Reflection Principle to a univalent function $\hat{\zeta}_{\theta_{n}}^{1}$ on $\hat{\Omega}^{1}$. Furthermore, the origin in $\hat{\Omega}^{1}$ is an interior point such that $\hat{\zeta}_{\theta_{n}}^{1}(0)=0$ and $\left(\hat{\zeta}_{\theta_{n}}^{1}\right)^{\prime}(0)>0$ for all $n$. The statement about the derivative follows since $\zeta_{\theta_{n}}^{1}$ maps points on the real axis to points on the real axis. Similarly, by reflecting around the point $2+\sqrt{3}$ we can obtain a sequence of univalent functions $\hat{\zeta}_{\theta_{n}}^{2}$ on an extended domain $\hat{\Omega}^{2}$ with $\hat{\zeta}_{\theta_{n}}^{2}(2+\sqrt{3})=1$ and $\left(\hat{\zeta}_{\theta_{n}}^{2}\right)^{\prime}(2+\sqrt{3})>0$ for all $n$.

Now, the regions $\zeta_{\theta_{n}}\left(\Omega^{j}\right)$ converge to $P_{-\pi / 6}^{j}, j=1,2$. This is because

$$
\operatorname{Ext}_{\Omega_{\theta_{n}}}\left(e_{4}, A_{\theta_{n}}\right), \operatorname{Ext}_{\Omega_{\theta_{n}}}\left(e_{2}, A_{\theta_{n}}\right) \rightarrow \infty \text { as } n \rightarrow \infty
$$

so that the curve $\zeta_{\theta_{n}}\left(A_{\theta_{n}}\right)$ must converge to the point $p$ (see figure 9 ). Therefore, we have from Theorem 2.2 that $\zeta_{\theta_{n}}^{j}$ converges to a limiting map $\zeta_{-\pi / 6}^{j}$ between the triangles $\Omega^{j}$ and $P_{-\pi / 6}^{j}, j=1,2$. Moreover, the convergence is uniform on compact subsets of the closure of $\Omega^{j}$ minus the edge $e_{3}^{1}$ (or $e_{3}^{\mathrm{u}}$ ) and the arc $A_{\theta}$.

To finish the proof, note that $\Pi(\theta)$ is given by

$$
\Pi(\theta)=I_{1}(\theta)+I_{2}(\theta)
$$

where the real integrals $I_{k}(\theta)$ are given by

$$
I_{1}(\theta)=\int_{0}^{1}\left(1-w^{2}\right) \zeta_{\theta}^{\prime}(w)^{2} d w>0
$$

and

$$
I_{2}(\theta)=\int_{1}^{2+\sqrt{3}}\left(1-w^{2}\right) \zeta_{\theta}^{\prime}(w)^{2} d w<0 .
$$


In particular, it is crucial here that

$$
V_{-\pi / 6}>1
$$

There are two cases to consider, and we begin with the case $p>0$. Here, we have that the angle between $e_{1}$ and $e_{3}^{1}$ on $\Omega^{1}$ (for $\theta=-\pi / 6$ ) is $120^{\circ}$, and this angle is mapped by $\zeta_{-\pi / 6}^{1}$ to an angle of $45^{\circ}$ on $P_{-\pi / 6}^{1}$. So, it follows that $\left(\zeta_{-\pi / 6}^{1}\right)^{\prime}(w)^{2}$ is not integrable at $V_{-\pi / 6}$. Thus, we have

$$
\int_{1}^{V_{-\pi / 6}}\left(1-w^{2}\right)\left(\zeta_{-\pi / 6}^{1}\right)^{\prime}(w)^{2} d w=-\infty
$$

and so we can choose $1<u_{0}<V_{-\pi / 6}$ such that

$$
I_{1}(-\pi / 6)+\int_{1}^{u_{0}}\left(1-w^{2}\right)\left(\zeta_{-\pi / 6}^{1}\right)^{\prime}(w)^{2} d w<0 .
$$

Because of the uniform convergence, we have that

$$
\begin{aligned}
& I_{1}\left(\theta_{n}\right)+\int_{1}^{u_{0}}\left(1-w^{2}\right)\left(\zeta_{\theta_{n}}^{1}\right)^{\prime}(w)^{2} d w \rightarrow I_{1}(-\pi / 6) \\
& \quad+\int_{1}^{u_{0}}\left(1-w^{2}\right)\left(\zeta_{-\pi / 6}^{1}\right)^{\prime}(w)^{2} d w
\end{aligned}
$$

as $\theta_{n} \rightarrow-\pi / 6$. Therefore, we may choose a positive integer $N_{1}$ such that

$$
I_{1}\left(\theta_{n}\right)+\int_{1}^{u_{0}}\left(1-w^{2}\right)\left(\zeta_{\theta_{n}}^{1}\right)^{\prime}(w)^{2} d w<0
$$

for any $n>N_{1}$. Finally, since

$$
I_{1}\left(\theta_{n}\right)+I_{2}\left(\theta_{n}\right) \leq I_{1}\left(\theta_{n}\right)+\int_{1}^{u_{0}}\left(1-w^{2}\right)\left(\zeta_{\theta_{n}}^{1}\right)^{\prime}(w)^{2} d w
$$

we have

$$
\Pi\left(\theta_{n}\right)<0, \text { for } n>N_{1}
$$

If $p=0$, then

$$
\zeta_{-\pi / 6}^{1} \equiv 0
$$

and

$$
\int_{V_{-\pi / 6}}^{2+\sqrt{3}}\left(1-w^{2}\right)\left(\zeta_{-\pi / 6}^{2}\right)^{\prime}(w)^{2} d w<0
$$


Thus, we can find points

$$
1<u_{1}<V_{-\pi / 6} \text { and } V_{-\pi / 6}<w_{1}<2+\sqrt{3}
$$

such that

$$
\int_{0}^{u_{1}}\left(1-w^{2}\right)\left(\zeta_{-\pi / 6}^{1}\right)^{\prime}(w)^{2} d w+\int_{w_{1}}^{2+\sqrt{3}}\left(1-w^{2}\right)\left(\zeta_{-\pi / 6}^{2}\right)^{\prime}(w)^{2} d w<0 .
$$

Therefore, because of the uniform convergence there exists a positive integer $N_{2}$ such that

$$
\int_{0}^{u_{1}}\left(1-w^{2}\right)\left(\zeta_{\theta_{n}}^{1}\right)^{\prime}(w)^{2} d w+\int_{w_{1}}^{2+\sqrt{3}}\left(1-w^{2}\right)\left(\zeta_{\theta_{n}}^{2}\right)^{\prime}(w)^{2} d w<0
$$

for any $n>N_{2}$. Since

$$
\begin{aligned}
I_{1}\left(\theta_{n}\right)+I_{2}\left(\theta_{n}\right) \leq & \int_{0}^{u_{1}}\left(1-w^{2}\right)\left(\zeta_{\theta_{n}}^{1}\right)^{\prime}(w)^{2} d w \\
& +\int_{w_{1}}^{2+\sqrt{3}}\left(1-w^{2}\right)\left(\zeta_{\theta_{n}}^{2}\right)^{\prime}(w)^{2} d w
\end{aligned}
$$

it follows that

$$
\Pi\left(\theta_{n}\right)<0, \quad n>N_{2}
$$

Therefore, in either case we may choose $\hat{n}$ such that $\hat{\theta}=\theta_{\hat{n}}$ satisfies

$$
\Pi(\hat{\theta})<0
$$

2.5.2. Adding a zig-zag At this point, the most obvious way to proceed would be to show the period is positive near the endpoint $\theta=\pi / 4$. Then, we could use the Intermediate value theorem to show there is some value of $\theta$ for which the period is zero. Such an argument would prove the existence of a soap film as in figure 1 spanning a tetrahedron. However, all calculations have indicated $\Pi(\theta)$ is also negative for values of $\theta$ near $\pi / 4$. Thus, to show the period is positive we must modify the boundary spanned by our soap film. We do this by introducing a $z i g$-zag into the tetrahedral edge $e_{3}$, where a zigzag consists of two horizontal, parallel line segments $H^{\mathrm{l}}$ and $H^{\mathrm{u}}$ connected by a non-horizontal line segment $e_{5}$ (see figure 10).

On the Gauss image, the introduction of a zig-zag has the following effect. First of all, by Proposition 2.3 we may choose $\Omega_{\hat{\theta}}$ so that $\Pi(\hat{\theta})<0$. 


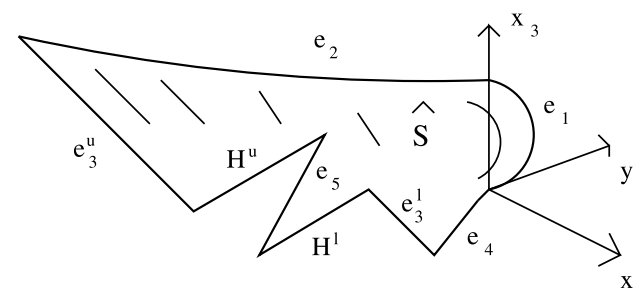

Figure 10: A fundamental piece $\hat{S}$ with an inserted zig-zag.

Next, we choose a horizontal segment $H^{1}$ whose Gauss image in contained in the line $\Lambda$ through the origin and the vertex

$$
v_{3}=\mathrm{e}^{\mathrm{i} \pi / 4}+\sqrt{2} \mathrm{e}^{\mathrm{i} \hat{\theta}} .
$$

After moving some distance along $\Lambda$ toward the origin, the segment $e_{5}$ is then chosen so that its Gauss image is contained in some circle $C_{5}^{t}$, where

$$
C_{5}^{t}=\partial D\left(\frac{t+\mathrm{i} t}{\sqrt{1-2 t^{2}}}, \frac{1}{\sqrt{1-2 t^{2}}}\right), \quad-1 / \sqrt{2}<t<0 .
$$

We will assume $t$ is fixed. Then, as with $e_{3}$ we introduce a branch point into the Gauss map along $e_{5}$, which separates $e_{5}$ on the Gauss image into two edges $e_{5}^{1}$ and $e_{5}^{\mathrm{u}}$. Finally, we return to $v_{3}$ along $\Lambda$, which introduces the second horizontal edge $H^{\mathrm{u}}$ (see figure 11). We thus have a new one-parameter family of Gauss image domains

$$
\left\{\Omega_{\hat{\theta}}^{s}\right\}, \quad 0<s<S_{\max }
$$

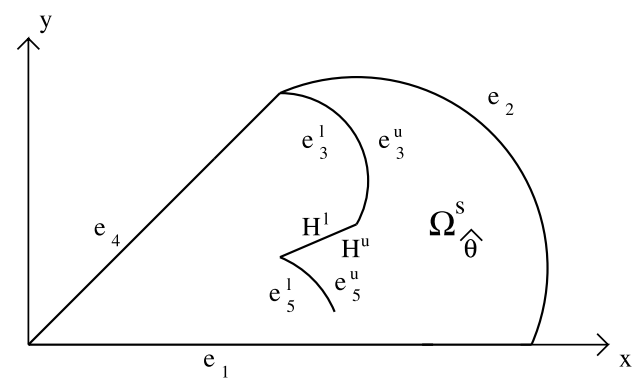

Figure 11: A Gauss image with an inserted zig-zag. 


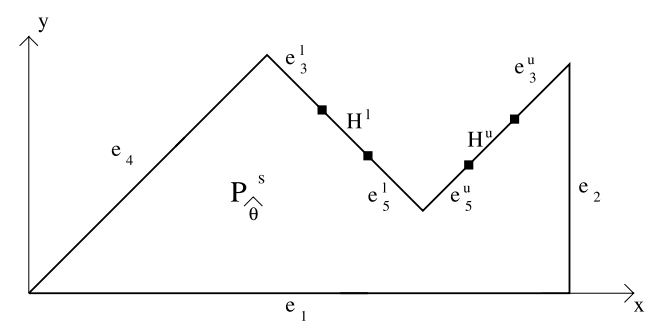

Figure 12: A developed image with an inserted zig-zag.

where $s$ is the distance from $v_{3}$ along $H^{1} \cup e_{5}^{1}$. If $s=0$, then

$$
\Omega_{\hat{\theta}}^{0}=\Omega_{\hat{\theta}}
$$

and the period function

$$
\Pi(\hat{\theta}, 0)<0
$$

As $s$ moves away from zero toward $S_{\text {max }}$, the horizontal segment $H^{1}$ appears. After some positive distance, the non-horizontal segment $e_{5}^{\mathrm{l}} \cup e_{5}^{\mathrm{u}}$ along with the second horizontal segment $H^{\mathrm{u}}$ are introduced into the boundary. Finally, the value $S_{\max }$ corresponds to the value of $s$ for which $v_{5}$ lies on the $x$-axis.

The effect of the zig-zag on the developed image $P_{\hat{\theta}}$ is shown in figure 12 . In particular, we have that the developing map $\zeta_{\hat{\theta}}^{s}$ should take $H^{\mathrm{l}}$ and $e_{5}^{\mathrm{l}}$ into lines parallel to $y=-x$ and it should take $H^{\mathrm{u}}$ and $e_{5}^{\mathrm{u}}$ into lines parallel to $y=$ $x$. The proof of the existence of this conformal, edge preserving map $\zeta_{\hat{\theta}}^{s}$ is the same as in Proposition 2.2: The edges $e_{3}^{\mathrm{l}}$ and $e_{3}^{\mathrm{u}}$ are simply replaced by $e_{3}^{\mathrm{l}} \cup$ $H^{\mathrm{l}} \cup e_{5}^{\mathrm{l}}$ and $e_{3}^{\mathrm{u}} \cup H^{\mathrm{u}} \cup e_{5}^{u}$, respectively. Thus, we obtain a parametrization

$$
X: \Omega_{\hat{\theta}}^{s} \rightarrow \mathbb{R}^{3}
$$

for each $s$. Furthermore, the zig-zag does not introduce any non-integrable singularities into $\left(d \zeta_{\hat{\theta}}^{s}\right)^{2}$, and so we have that the image

$$
\overline{X\left(\Omega_{\hat{\theta}}^{s}\right)} \text { is compact. }
$$

Before analyzing the period $\Pi(\hat{\theta}, s)$ for these maps, we need to check the parameterizations along the new edges $H^{\mathrm{l}}, H^{\mathrm{u}}, e_{5}^{\mathrm{l}}$ and $e_{5}^{\mathrm{u}}$. 
Beginning with $H^{\mathrm{l}}$, we parameterize $\Lambda$ on $\Omega_{\hat{\theta}}^{s}$ from

$$
v_{H}^{1}=H^{1} \cap e_{5}^{1} \text { to } v_{3}^{1}=e_{3}^{1} \cap H^{1}
$$

by $z_{H}(w)=w \mathrm{e}^{\mathrm{i} \phi}$, where $w>0$ and $\phi$ is the angle $\Lambda$ makes with the $x$ axis. Here, we have $\dot{z}_{H}(w)=\mathrm{e}^{\mathrm{i} \phi}$ and $d \zeta\left(\dot{z}_{H}\right)^{2}=-\mathrm{i}\left|d \zeta\left(\dot{z}_{H}\right)\right|^{2}$. Computing, we obtain

$$
\begin{aligned}
d X_{1}\left(\dot{z}_{H}\right) & =-\frac{\left|d \zeta\left(\dot{z}_{H}\right)\right|^{2}}{2} \operatorname{Re}\left(\mathrm{ie}^{-\mathrm{i} \phi}\left(1-w^{2} \mathrm{e}^{\mathrm{i} 2 \phi}\right)\right) \\
& =-\frac{\left|d \zeta\left(\dot{z}_{H}\right)\right|^{2}}{2}\left(1+w^{2}\right) \sin \phi<0
\end{aligned}
$$

Continuing, we have

$$
\begin{aligned}
d X_{2}\left(\dot{z}_{H}\right) & =\frac{\left|d \zeta\left(\dot{z}_{H}\right)\right|^{2}}{2} \operatorname{Re}\left(\mathrm{e}^{-\mathrm{i} \phi}\left(1+w^{2} \mathrm{e}^{\mathrm{i} 2 \phi}\right)\right) \\
& =\frac{\left|d \zeta\left(\dot{z}_{H}\right)\right|^{2}}{2}\left(1+w^{2}\right) \cos \phi>0
\end{aligned}
$$

For the $x_{3}$ component, we have

$$
d X_{3}\left(\dot{z}_{H}\right)=-\left|d \zeta\left(\dot{z}_{H}\right)\right|^{2} \operatorname{Re}(\mathrm{i} w)=0 .
$$

From Equations (2.35) to (2.37) we have that the vector $d X$ is given by

$$
\left\langle d X_{1}\left(\dot{z}_{H}\right), d X_{2}\left(\dot{z}_{H}\right), d X_{3}\left(\dot{z}_{H}\right)\right\rangle=\frac{\left|d \zeta\left(\dot{z}_{H}\right)\right|^{2}}{2}\left(1+w^{2}\right)\langle-\sin \phi, \cos \phi, 0\rangle .
$$

Thus, we have shown that $X$ maps $H^{1}$ monotonically onto a horizontal line segment which makes an angle of $\pi / 2-\phi$ with the $x$-axis. Furthermore, we have that $X_{2}$ is increasing as the parameter moves along $H^{1}$ from $v_{H}^{1}$ to $v_{3}^{1}$.

If we parameterize $H^{\mathrm{u}}$ in the same direction as $H^{\mathrm{l}}$, then the parametrization is the same but we have $d \zeta^{2}=\mathrm{i}|d \zeta|^{2}$. So, the calculations in this case will give

$$
d X=\frac{|d \zeta|^{2}}{2}\left(1+w^{2}\right)\langle\sin \phi,-\cos \phi, 0\rangle .
$$

Thus, we have that $X$ also maps $H^{\mathrm{u}}$ monotonically onto a line segment which makes an angle of $\pi / 2-\phi$ with the $x$-axis. Furthermore, we have 
that $X_{2}$ is decreasing as the parameter moves from

$$
v_{H}^{\mathrm{u}}=H^{\mathrm{u}} \cap e_{5}^{\mathrm{u}} \text { to } v_{3}^{\mathrm{u}}=e_{3}^{\mathrm{u}} \cap H^{\mathrm{u}}
$$

Next, we parameterize $e_{5}^{1}$ in the counterclockwise direction from $v_{5}$ to $v_{H}^{\mathrm{l}}$ by $z_{5}(w)=(t+\mathrm{i} t) / \sqrt{1-2 t^{2}}+\mathrm{e}^{\mathrm{i} w} / \sqrt{1-2 t^{2}}$ (recall that $\left.t<0\right)$. Here, we have $\dot{z}_{5}(w)=\mathrm{ie} \mathrm{i}^{\mathrm{i} w} / \sqrt{1-2 t^{2}}$, and $d \zeta\left(\dot{z}_{5}\right)^{2}=-\mathrm{i}\left|d \zeta\left(\dot{z}_{5}\right)\right|^{2}$. Computing, we have

$$
\begin{aligned}
d X_{1}\left(\dot{z}_{5}\right) & =-\frac{\left|d \zeta\left(\dot{z}_{5}\right)\right|^{2}}{2 \sqrt{1-2 t^{2}}} \operatorname{Re}\left(\mathrm{e}^{-\mathrm{i} w}\left(1-2 t^{2}-\mathrm{e}^{\mathrm{i} 2 w}-\mathrm{i} 2 t^{2}-2 t \mathrm{e}^{\mathrm{i} w}-\mathrm{i} 2 t \mathrm{e}^{\mathrm{i} w}\right)\right) \\
& =\frac{t\left|d \zeta\left(\dot{z}_{5}\right)\right|^{2}}{\sqrt{1-2 t^{2}}}(1+t \cos w+t \sin w)<0
\end{aligned}
$$

Continuing, we have

$$
\begin{aligned}
d X_{2}\left(\dot{z}_{5}\right) & =-\frac{\left|d \zeta\left(\dot{z}_{5}\right)\right|^{2}}{2 \sqrt{1-2 t^{2}}} \operatorname{Re}\left(\mathrm{ie}^{-\mathrm{i} w}\left(1-2 t^{2}+\mathrm{e}^{\mathrm{i} 2 w}+\mathrm{i} 2 t^{2}+2 t \mathrm{e}^{\mathrm{i} w}+\mathrm{i} 2 t \mathrm{e}^{\mathrm{i} w}\right)\right) \\
& =\frac{t\left|d \zeta\left(\dot{z}_{5}\right)\right|^{2}}{\sqrt{1-2 t^{2}}}(1+t \cos w+t \sin w)<0 .
\end{aligned}
$$

For the $x_{3}$ component, we have

$$
\begin{aligned}
d X_{3}\left(\dot{z}_{5}\right) & =-\left|d \zeta\left(\dot{z}_{5}\right)\right|^{2} \operatorname{Re}\left(\mathrm{e}^{-\mathrm{i} w}\left(t+\mathrm{i} t+\mathrm{e}^{\mathrm{i} w}\right)\right) \\
& =-\left|d \zeta\left(\dot{z}_{5}\right)\right|^{2}(1+t \cos w+t \sin w)<0
\end{aligned}
$$

From Equations (2.38) to (2.40) we have

$$
\begin{aligned}
\left\langle d X_{1}\left(\dot{z}_{5}\right), d X_{2}\left(\dot{z}_{5}\right), d X_{3}\left(\dot{z}_{5}\right)\right\rangle= & \frac{\left|d \zeta\left(\dot{z}_{5}\right)\right|^{2}}{\sqrt{1-2 t^{2}}}(1+t \cos w+t \sin w) \\
& \left\langle t, t,-\sqrt{1-2 t^{2}}\right\rangle
\end{aligned}
$$

Thus, we have that $X$ maps $e_{5}^{1}$ monotonically onto a Euclidean line segment in the direction $\left\langle t, t,-\sqrt{1-2 t^{2}}\right\rangle$. Furthermore, we have that $X_{3}$ is decreasing as the parameter moves from $v_{5}$ to $v_{H}^{\mathrm{l}}$. This verifies that $H^{\mathrm{u}}$ lies above $H^{\mathrm{l}}$.

If we parameterize $e_{5}^{\mathrm{u}}$ in the counterclockwise direction, then the parametrization is the same as for $e_{5}^{1}$ but we have $d \zeta^{2}=\mathrm{i}|d \zeta|^{2}$. So, the 
calculations in this case will give

$$
d X=\frac{|d \zeta|^{2}}{\sqrt{1-2 t^{2}}}(1+t \cos w+t \sin w)\left\langle-t,-t, \sqrt{1-2 t^{2}}\right\rangle .
$$

Together with the findings for $e_{5}^{1}$, we have that $X$ maps $e_{5}^{1} \cup e_{5}^{\mathrm{u}}$ monotonically onto a line segment in the direction $\left\langle t, t, \sqrt{1-2 t^{2}}\right\rangle$. We have now verified that we obtain the desired zig-zag on the image $X\left(\Omega_{\hat{\theta}}^{s}\right)$.

2.5.3. Positive period We now consider the case where $s \rightarrow S_{\max }$ and prove the following proposition. The proof is similar to that of Proposition 2.3.

Proposition 2.4. There is a value $0<\hat{s}<S_{\max }$ such that $\Pi(\hat{\theta}, \hat{s})>0$.

Proof. First of all, note that

$$
\operatorname{Ext}_{\Omega_{\hat{\theta}}}^{s}\left(e_{5}^{\mathrm{u}}, e_{1}\right) \rightarrow 0 \text { as } s \rightarrow S_{\max }
$$

and so the corresponding behavior in $P_{\hat{\theta}}^{s}$ must be that the vertex $\zeta_{\hat{\theta}}^{s}\left(v_{5}\right)$ approaches the edge $e_{1}$. Thus, because of compactness there is a sequence $s_{n} \rightarrow S_{\max }$ and a point $0 \leq p \leq 1$ such that

$$
\zeta_{\hat{\theta}}^{s_{n}}\left(v_{5}\right) \rightarrow p \text { as } n \rightarrow \infty
$$

Let $P_{\hat{\theta}}^{S_{\max }}$ be a (degenerate) Euclidean polygon with edges oriented and labeled as in figure 12 that is determined by the normalizations $e_{1} \cap e_{4}=0$, $\left|e_{1}\right|=1$ and the point $p$. Such a degenerate polygon consists of two triangles

$$
P_{\hat{\theta}}^{S_{\max }, 1} \text { and } P_{\hat{\theta}}^{S_{\max }, 2},
$$

where $P_{\hat{\theta}}^{S_{\max }}=P_{\hat{\theta}}^{S_{\max }, 1}$ if $p=1$ and $P_{\hat{\theta}}^{S_{\max }}=P_{\hat{\theta}}^{S_{\max }, 2}$ if $p=0$. To apply Theorem 2.2 , we separate $\Omega_{\hat{\theta}}^{s}$ for $s$ near $S_{\max }$ into two regions $\Omega_{\hat{\theta}}^{S_{\max }, 1}$ and $\Omega_{\hat{\theta}}^{S_{\max }, 2}$ via the arc $A_{s}$ of $C_{5}^{t}$ connecting $v_{5}$ to the positive $x$-axis. The point of $C_{5}^{t}$ that lies on the positive $x$-axis is

$$
V_{S_{\max }}=\frac{t+\sqrt{1-t^{2}}}{\sqrt{1-2 t^{2}}}
$$

What is important to note here is that

$$
V_{S_{\max }}<1
$$

which follows from the fact that $-1 / \sqrt{2}<t<0$. 
With this separation, the regions $\zeta_{\hat{\theta}}^{s}\left(\Omega_{\hat{\theta}}^{S_{\max }, j}\right)$ converge to $P_{\hat{\theta}}^{S_{\max }, j}, j=$ 1,2. Arguing as in Proposition 2.3, it follows from Theorem 2.2 that $\zeta_{\hat{\theta}}^{s, j}$, which denotes the restriction of $\zeta_{\hat{\theta}}^{s}$ to $\Omega_{\hat{\theta}}^{S_{\max }, j}$, converges to a map $\zeta_{\hat{\theta}}^{S_{\max }, j}$ between the regions $\Omega_{\hat{\theta}}^{S_{\max }, j}$ and $P_{\hat{\theta}}^{S_{\max }, j}, j=1,2$. Moreover, the convergence is uniform on compact subsets of the closure of $\Omega_{\hat{\theta}}^{S_{\max }, j}$ minus the edge $e_{3}^{1} \cup$ $H^{\mathrm{l}} \cup e_{5}^{\mathrm{l}} \cup A_{s}$ (or $\left.e_{3}^{\mathrm{u}} \cup H^{\mathrm{u}} \cup e_{5}^{\mathrm{u}} \cup A_{s}\right)$.

To finish the proof, recall that $\Pi(\hat{\theta}, s)$ is given by

$$
\Pi(\hat{\theta}, s)=I_{1}(\hat{\theta}, s)+I_{2}(\hat{\theta}, s)
$$

where the real integrals $I_{k}(\hat{\theta}, s)$ are given by

$$
I_{1}(\hat{\theta}, s)=\int_{0}^{1}\left(1-w^{2}\right)\left(\zeta_{\hat{\theta}}^{s}\right)^{\prime}(w)^{2} d w>0
$$

and

$$
I_{2}(\hat{\theta})=\int_{1}^{2+\sqrt{3}}\left(1-w^{2}\right)\left(\zeta_{\hat{\theta}}^{s}\right)^{\prime}(w)^{2} d w<0 .
$$

It is crucial here that

$$
V_{S_{\max }}<1
$$

There are two cases to consider, and we begin with the case $p<1$. Here, we have that the angle between $e_{1}$ and $e_{5}^{\mathrm{u}}$ on $\Omega_{\hat{\theta}}^{S_{\max }, 2}$ is

$$
\arccos (t)>\pi / 2
$$

and this angle is mapped by $\zeta_{\hat{\theta}}^{S_{\max }, 2}$ to an angle of $\pi / 4$ on $P_{\hat{\theta}}^{S_{\max }, 2}$. So, it follows that $\left(\zeta_{\hat{\theta}}^{S_{\max }, 2}\right)^{\prime}(w)^{2}$ is not integrable at $V_{S_{\max }}$. Thus, we have

$$
\int_{V_{S_{\max }}}^{1}\left(1-w^{2}\right)\left(\zeta_{\hat{\theta}}^{S_{\max }, 2}\right)^{\prime}(w)^{2} d w=\infty
$$

and so we can choose $V_{S_{\max }}<u_{0}<1$ such that

$$
\int_{u_{0}}^{1}\left(1-w^{2}\right)\left(\zeta_{\hat{\theta}}^{S_{\max }, 2}\right)^{\prime}(w)^{2} d w+I_{2}\left(\hat{\theta}, S_{\max }\right)>0
$$


Because of the uniform convergence, we have that

$$
\begin{aligned}
& \int_{u_{0}}^{1}\left(1-w^{2}\right)\left(\zeta_{\hat{\theta}}^{s_{n}, 2}\right)^{\prime}(w)^{2} d w+I_{2}\left(\hat{\theta}, s_{n}\right) \\
& \quad \longrightarrow \int_{u_{0}}^{1}\left(1-w^{2}\right)\left(\zeta_{\hat{\theta}}^{S_{\max }, 2}\right)^{\prime}(w)^{2} d w+I_{2}\left(\hat{\theta}, S_{\max }\right)
\end{aligned}
$$

as $n \rightarrow \infty$. Therefore, we may choose a positive integer $N_{1}$ such that

$$
\int_{u_{0}}^{1}\left(1-w^{2}\right)\left(\zeta_{\hat{\theta}}^{s_{n}, 2}\right)^{\prime}(w)^{2} d w+I_{2}\left(\hat{\theta}, s_{n}\right)>0
$$

for any $n>N_{1}$. Finally, since

$$
I_{1}\left(\hat{\theta}, s_{n}\right)+I_{2}\left(\hat{\theta}, s_{n}\right) \geq \int_{u_{0}}^{1}\left(1-w^{2}\right)\left(\zeta_{\hat{\theta}}^{s_{n}, 2}\right)^{\prime}(w)^{2} d w+I_{2}\left(\hat{\theta}, s_{n}\right)
$$

we have

$$
\Pi\left(\hat{\theta}, s_{n}\right)>0 \text {, for } n>N_{1}
$$

If $p=1$, then

$$
\zeta_{\hat{\theta}}^{S_{\max }, 2} \equiv 0
$$

and

$$
\int_{0}^{V_{S_{\max }}}\left(1-w^{2}\right)\left(\zeta_{\hat{\theta}}^{S_{\max }, 1}\right)^{\prime}(w)^{2} d w>0
$$

Thus, we can find points

$$
0<w_{1}<V_{S_{\max }} \text { and } V_{S_{\max }}<u_{1}<1
$$

such that

$$
\int_{0}^{w_{1}}\left(1-w^{2}\right)\left(\zeta_{\hat{\theta}}^{S_{\max }, 1}\right)^{\prime}(w)^{2} d w+\int_{u_{1}}^{2+\sqrt{3}}\left(1-w^{2}\right)\left(\zeta_{\hat{\theta}}^{S_{\max }, 2}\right)^{\prime}(w)^{2} d w>0
$$

Therefore, because of the uniform convergence there exists a positive integer $N_{2}$ such that

$$
\int_{0}^{w_{1}}\left(1-w^{2}\right)\left(\zeta_{\hat{\theta}}^{s_{n}, 1}\right)^{\prime}(w)^{2} d w+\int_{u_{1}}^{2+\sqrt{3}}\left(1-w^{2}\right)\left(\zeta_{\hat{\theta}}^{s_{n}, 2}\right)^{\prime}(w)^{2} d w>0
$$


for any $n>N_{2}$. Since

$$
\begin{aligned}
I_{1}\left(\hat{\theta}, s_{n}\right)+I_{2}\left(\hat{\theta}, s_{n}\right) \geq & \int_{0}^{w_{1}}\left(1-w^{2}\right)\left(\zeta_{\hat{\theta}}^{s_{n}, 1}\right)^{\prime}(w)^{2} d w \\
& +\int_{u_{1}}^{2+\sqrt{3}}\left(1-w^{2}\right)\left(\zeta_{\hat{\theta}}^{s_{n}, 2}\right)^{\prime}(w)^{2} d w,
\end{aligned}
$$

it follows that

$$
\Pi\left(\hat{\theta}, s_{n}\right)>0, n>N_{2} .
$$

Therefore, in either case we may choose $\hat{n}$ such that $\hat{s}=s_{\hat{n}}$ satisfies

$$
\Pi(\hat{\theta}, \hat{s})>0 .
$$

From Propositions 2.3, 2.4 and the Intermediate value theorem it follows that there is some value $0<s_{0}<\hat{s}$ such that

$$
\Pi\left(\hat{\theta}, s_{0}\right)=0 .
$$

Moreover, this holds for each $-1 / \sqrt{2}<t<0$.

\subsection{Extension to an immersed soap film}

The parametrization $X$ on $\Omega_{\hat{\theta}}^{s_{0}}$ is a conformal, minimal immersion which has the desired boundary and satisfies the period condition. If we reflect $\Omega_{\hat{\theta}}^{s_{0}}$ and $P_{\hat{\theta}}^{s_{0}}$ around the origin (see figure 13), we obtain two conformal disks $\hat{\Omega}_{\hat{\theta}}^{s_{0}}$ and $\hat{P}_{\hat{\theta}}^{s_{0}}$, and by the Schwarz Reflection Principle we also extend the map $\zeta_{\hat{\theta}}^{s_{0}}$ to a map

$$
\hat{\zeta}_{\hat{\theta}}^{s_{0}}: \hat{\Omega}_{\hat{\theta}}^{s_{0}} \rightarrow \hat{P}_{\hat{\theta}}^{s_{0}}
$$

This extends the immersion $X$ to a map

$$
\Phi=\left(\Phi_{1}, \Phi_{2}, \Phi_{3}\right): \hat{\Omega}_{\hat{\theta}}^{s_{0}} \rightarrow \mathbb{R}^{3}
$$

given by Weierstrass data

$$
g(z)=z \quad \text { and } \quad d h=\frac{g\left(d \hat{\zeta}_{\hat{\theta}}^{s_{0}}\right)^{2}}{d g} .
$$

The image of $X$ generates the image of $\Phi$ as follows:

1. Reflecting $\Omega_{\hat{\theta}}^{s_{0}}$ through the edge $e_{1}$ reflects $X\left(\Omega_{\hat{\theta}}^{s_{0}}\right)$ through the edge $X\left(e_{1}\right)$. 


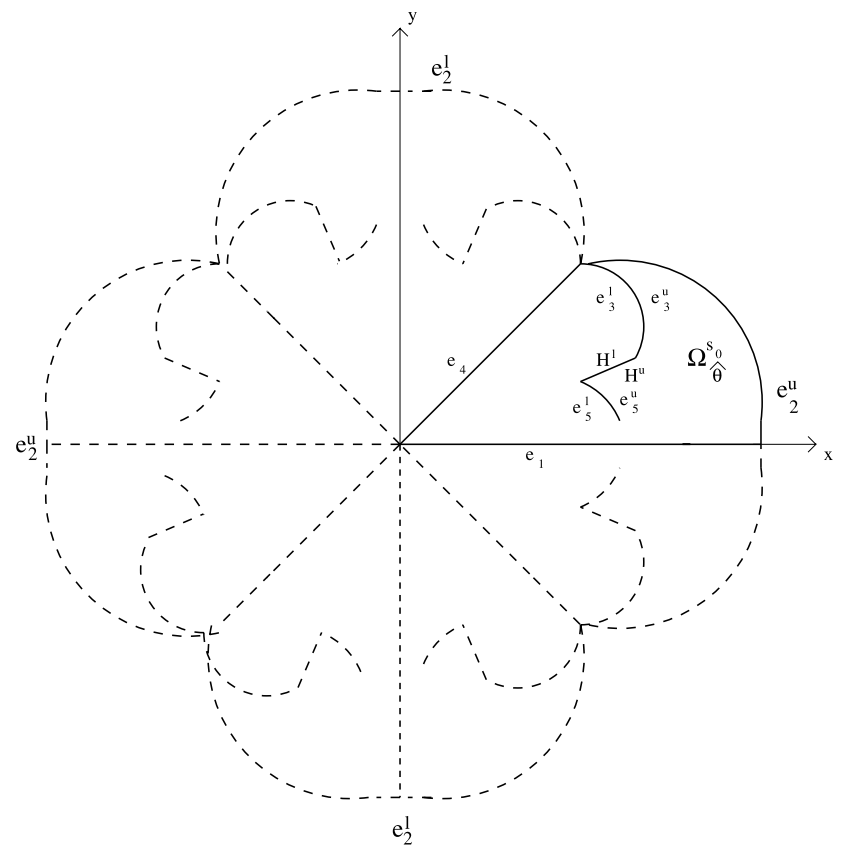

Figure 13: The punctured torus is obtained by identifying opposite edges of $\hat{\Omega}_{\hat{\theta}}^{s_{0}}$.

2. Reflecting $\Omega_{\hat{\theta}}^{s_{0}}$ through the edge $e_{4}$ rotates $X\left(\Omega_{\hat{\theta}}^{s_{0}}\right) 180^{\circ}$ around the edge $X\left(e_{4}\right)$.

Thus, since the period condition is satisfied, the map $\Phi$ is well defined on the punctured torus $\mathcal{T}$ obtained by identifying the interiors of the left and right edges $e_{2}^{\mathrm{u}}$ via the identification $z \rightarrow-\bar{z}$ as well as the interiors of the top and bottom edges $e_{2}^{l}$ via the identification $z \rightarrow \bar{z}$ (see figure 13).

We next attach two disks to the punctured torus as follows. The first disk $D_{1}$ we choose to be bounded by the right edge $e_{2}^{\mathrm{u}}$ of $\hat{\Omega}_{\hat{\theta}}^{s_{0}}$ and the vertical line segment between $v_{23}$ and $\bar{v}_{23}$. We then attach $D_{1}$ to the torus $\mathcal{T}$ by identifying the edges $e_{2}^{\mathrm{u}}$ via the identification $z \rightarrow z$. The map $\Phi$ can then be extended to $D_{1}$ by mapping a point $z$ in $D_{1}$ to the point in the $y x_{3}$-plane such that

$$
\Phi_{2}(z)=\Phi_{2}\left(\xi_{0}\right)
$$

and

$$
\frac{\Phi_{3}(z)-\Phi_{3}\left(\xi_{0}\right)}{\Phi_{3}\left(v_{23}\right)-\Phi_{3}\left(\xi_{0}\right)}=\frac{\xi_{0}-z}{\xi_{0}-\xi_{1}}
$$


where $\xi_{0} \in e_{2}^{\mathrm{u}}$ is such that $\operatorname{Im} \xi_{0}=\operatorname{Im} z$ and $\xi_{1}$ is such that $\operatorname{Re} \xi_{1}=\operatorname{Re} v_{23}$ and $\operatorname{Im} \xi_{1}=\operatorname{Im} z$. Similarly, we can extend $\Phi$ on the disk $D_{2}$ chosen to be bounded by the top edge $e_{2}^{1}$ of $\hat{\Omega}_{\hat{\theta}}^{s_{0}}$ and the horizontal line segment between $v_{34}$ and $-\bar{v}_{34}$.

We have thus constructed a set $\mathcal{X}$ which is the union of the punctured torus $\mathcal{T}$ and the disks $D_{1}$ and $D_{2}$ modulo the identifications mentioned above, where for $D_{1}$ we include the interior of $e_{2}^{\mathrm{u}}$ but not the vertical segment between $v_{23}$ and $\bar{v}_{23}$ and for $D_{2}$ we include the interior of $e_{2}^{1}$ but not the horizontal segment between $v_{34}$ and $-\bar{v}_{34}$. We have also constructed a map

$$
\Phi: \mathcal{X} \rightarrow \mathbb{R}^{3} \text {. }
$$

Except for the origin, it follows immediately that $\Phi$ is a minimal immersion on the interior of $\hat{\Omega}_{\hat{\theta}}^{s_{0}} \cup D_{1} \cup D_{2}$. At the origin, the Gauss map $g(z)=z$ has a zero of order 1 . Since $\left(\zeta_{\hat{\theta}}^{s_{0}}\right)^{\prime}(0)>0$, we have that $d h=z\left(\zeta_{\hat{\theta}}^{s_{0}}\right)^{\prime}(z)^{2}$ also has a zero of order 1 at the origin. Thus, by the Weierstrass Representation Theorem the map $\Phi$ is a minimal immersion at the origin. If $z$ is a point in the interior of $e_{2}^{\mathrm{u}}$, then we can find a neighborhood $U$ which is the intersection of an open disk with the closure of $\hat{\Omega}_{\hat{\theta}}^{s_{0}}$ such that $U$ contains only points in the interior of $\hat{\Omega}_{\hat{\theta}}^{s_{0}}$ or the interior of $e_{2}^{u}$. Furthermore, we can choose such a neighborhood $U$ so that $\Phi$ restricted to this neighborhood can be extended via the Schwarz Reflection Principle to an immersion on a $\mathbb{C}$-neighborhood of $z$. Then, taking $\mathcal{U}$ to be the union modulo identifications of $U, \tilde{U}=U \subset D_{1}$, and $-\bar{U} \ni-\bar{z}$, we have that $\Phi(\mathcal{U})$ consists of three minimal surfaces meeting along a curve at $120^{\circ}$. Similarly, for each point in the interior of $e_{2}^{1}$ we can find a neighborhood $\mathcal{U}$ such that $\Phi(\mathcal{U})$ consists of three minimal surfaces meeting along a curve at $120^{\circ}$.

\section{References}

[1] L. Ahlfors, Conformal invariants: topics in geometric function theory, McGraw-Hill Book Co., New York, 1973.

[2] M. Do Carmo, Differential geometry of curves and surfaces, Prentice Hall, Paris, 1976.

[3] C. Carathéodory, Conformal representation, Dover Publications, Inc., New York, 1998.

[4] D. Hoffman and H. Karcher, Complete embedded minimal surfaces of finite total curvature, in Encyclopedia of Mathematics, Osserman, R. ed., Springer Verlag, Berlin, 1997, 5-93. 
[5] G. Lawlor and F. Morgan, Paired calibrations applied to soap films, immiscible fluids, and surfaces or networks minimizing other norms, Pacific J. Math. 166(1) (1994), 55-83.

[6] J. Taylor, The structure of singularities in soap-bubble-like and soapfilm-like minimal surfaces, Ann. Math. 103 (1976), 489-539.

[7] M. Weber and M. Wolf, Teichmüller theory and handle addition for minimal surfaces, Ann. Math. 156(3) (2002), 713-795.

Department of Mathematics

HARDING UNIVERSITY

HU Box 10764

SEARCY

AR 72149

USA

E-mail address: rhuff3@gmail.com

RECEIVED JANUARY 27, 2010 
